\title{
Lumbar Intradural Neurenteric Cyst: A Rare Pathology in an Unusual Location
}

\author{
Sana Basseri, John P. Rossiter, M. Christopher Wallace, Omar Islam, \\ Donatella Tampieri, Benjamin Y.M. Kwan
}

Keywords: Spinal cord, MRI, Congenital Malformation

doi: $10.1017 / \operatorname{cjn} .2020 .148$

Can J Neurol Sci. 2021; 48: 116-117

A previously healthy 48 -year-old female presented to the emergency department with a 2-week history of low back pain, progressive lower extremities weakness, and right leg numbness. There were no bowel or bladder dysfunction symptoms. Spine magnetic resonance imaging (MRI) showed an intradural cystic lesion dorsal to the spinal cord at the level of L1 measuring $1.6 \times 2.1 \times 4.1 \mathrm{~cm}$, which was T1 hypointense and T2 hyperintense, with a small soft tissue component and no gadolinium enhancement (Figure 1). A small lipomatous component was also noted. There were no associated vertebral anomalies. The patient underwent a T12-L2 laminectomy and cyst resection, which was subtotal due to the cyst adherence to the conus medullaris. Histopathology showed characteristic features of a neurenteric cyst, with respiratory-type epithelium in the cyst wall (Figure 2). Eight months later, follow-up MRI showed no evidence of recurrence. The patient reported improved sensation in the lower extremities; however, there was some residual weakness predominantly in the proximal hip flexors bilaterally.

Spinal intradural extramedullary neurenteric cysts are rare, making up to $0.7 \%-1.3 \%$ of all spinal tumors, with a propensity for cervical and upper thoracic regions. ${ }^{1,2}$ Here, we present a case of a neurenteric cyst in an uncommon location at the L1 vertebral level. Neurenteric cysts are hypothesized to result from abnormal partitioning of the foregut endoderm from the notochordal plate during early embryogenesis. ${ }^{1}$ Histopathologically, a neurenteric cyst is lined by cuboidal or columnar epithelial cells which may demonstrate features of the respiratory or gastrointestinal tract, such as cilia, mucous glands, and/or goblet cells. ${ }^{2}$ This accounts for alternative terminologies in the literature including "endodermal" cyst or "bronchogenic" cyst when lined by respiratory epithelium, such as in our case.

The differential for intradural extramedullary lesions is broad with meningioma, schwannoma, and neurofibroma representing the most common lesions. ${ }^{3}$ Non-neoplastic intradural extramedullary spinal mass lesions include lipoma, dermoid, epidermoid, and arachnoid cysts, and rarely neurenteric cysts. ${ }^{2}$ The differential can sometimes be narrowed based on characteristic features on MRI, which is the modality of choice for distinguishing such lesions. The most common MRI finding for spinal neurenteric cyst is a homogeneous lesion that appears hypointense on $\mathrm{T} 1$ and hyperintense on $\mathrm{T} 2$ and does not enhance following administration of gadolinium contrast agent. ${ }^{1,2,4}$ However, variable $\mathrm{T} 1$ and $\mathrm{T} 2$ intensity have been described depending on the protein content of the cyst. Cases reported in the literature also occasionally demonstrate a lipomatous component on MRI, as seen in our case. ${ }^{4}$ Given the variable appearance and non-specific clinical presentation, histopathology is required for definitive diagnosis. Spinal neurenteric cysts may be associated with congenital
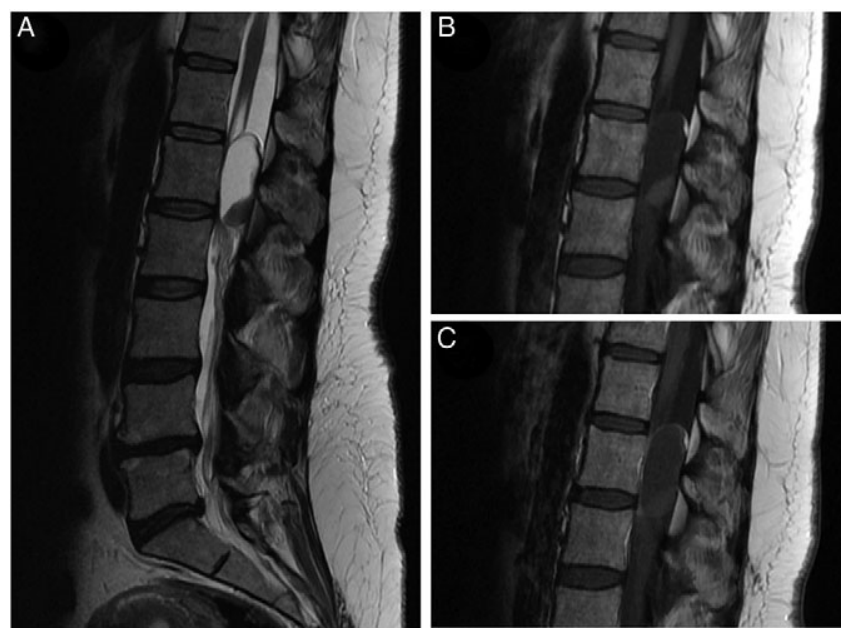

Figure 1: MRI images revealed an intradural lesion measuring $1.6 \times 2.1 \times 4.1 \mathrm{~cm}(A P \times T R \times C C)$ dorsal to the L1 vertebral body, demonstrating hyperintense signal on T2-weighted imaging shown in sagittal plane (A). Comparison of pre-gadolinium T1-weighted imaging $(\boldsymbol{B})$ and post-gadolinium T1-weighted imaging $(\boldsymbol{C})$ demonstrated no enhancement of the lesion. A small soft tissue component is seen posteroinferiorly, and a tiny focus of fat with chemical shift artifact is noted on $T 2$ at the posterosuperior corner of the lesion $(\boldsymbol{A}, \boldsymbol{B})$.

From the Department of Diagnostic Radiology, Queen's University, Kingston, Ontario, Canada (SB, OI, DT, BYMK); Department of Pathology and Molecular Medicine, Queen's University, Kingston, Ontario, Canada (JPR); and Department of Surgery, Division of Neurosurgery, Queen's University, Kingston, Ontario, Canada (MCW) Received June 22, 2020. Date of Acceptance July 1, 2020.

Correspondence to: Sana Basseri, Department of Diagnostic Radiology, Kingston General Hospital, 76 Stuart Street, Kingston, Ontario, K7L 2V7, Canada. Email: 18sb50@queensu.ca 
A

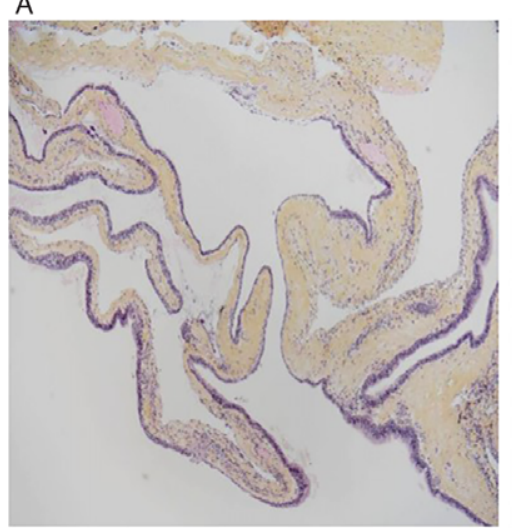

$\mathrm{B}$

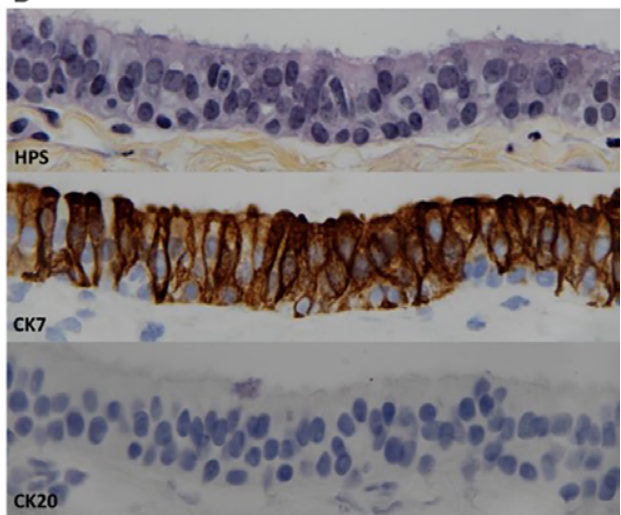

Figure 2: (A) Medium-power view of the partially resected cyst wall stained with hematoxylin phloxine saffron (HPS). (B) Cyst wall demonstrating pseudostratified ciliated columnar epithelial cells (respiratory type) that are strongly immunoreactive for intermediate filament cytokeratin CK7 and immuno-negative for CK20. Given the respiratory-type epithelium, the cyst could be termed a bronchogenic cyst.

vertebral anomalies such as Klippel-Feil, anterior spina bifida, butterfly vertebrae, and hemivertebrae. ${ }^{5}$ While they are often indolent, spinal neurenteric cysts may become symptomatic with cord compression. ${ }^{2,4}$ When possible, total surgical resection is the most effective management option to prevent recurrence. ${ }^{1,6}$

\section{CONFLICT OF INTEREST}

The authors have no conflicts of interest or financial support to disclose.

\section{Statement of Authorship}

We confirm that each author has participated sufficiently in this submission, taking public responsibility for its content, and has approved this submission.

SB organized the images, performed the literature review, and prepared the manuscript. JPR reviewed the pathology and revised the manuscript for intellectual content. MCW revised the manuscript for intellectual content. OI reviewed the radiological findings and revised the manuscript for intellectual content. DT reviewed the radiological findings and revised the manuscript for intellectual content. BYMK designed and conceptualized study, reviewed the radiological images, and revised the manuscript for intellectual content.

\section{REFERENCES}

1. Weng JC, Ma JP, Hao SY, et al. Intradural extramedullary bronchogenic cyst: clinical and radiologic characteristics, surgical outcomes, and literature review. World Neurosurg. 2018;109: e571-80.

2. Baek WK, Lachkar S, Iwanaga J, et al. Comprehensive review of spinal neurenteric cysts with a focus on histopathological findings. Cureus. 2018;10(9):e3379.

3. Koeller KK, Shih RY. Intradural extramedullary spinal neoplasms: radiologic-pathologic correlation. Radiographics. 2019;39(2): 468-90.

4. Ma X, Li W, Niu C, et al. Intraspinal bronchogenic cyst: series of case reports and literature review. J Spinal Cord Med. 2017; 40(2):141-6.

5. Can A, Dos Santos Rubio EJ, Jasperse B, Verdijk RM, Harhangi BS. Spinal Neurenteric Cyst in Association with Klippel-Feil Syndrome: case report and literature review. World Neurosurg. 2015;84(2):592. e9-14.

6. Garg N, Sampath S, Yasha TC, et al. Is total excision of spinal neurenteric cysts possible? Br J Neurosurg. 2008;22(2):241-51. 